\title{
Convergence of the compensated split-step $\theta$-method for nonlinear jump-diffusion systems
}

\author{
Jianguo $\operatorname{Tan}^{1 *}$ and Weiwei Men²
}

*Correspondence: tanjg@tju.edu.cn 1 Department of Mathematics, Tianjin Polytechnic University, Tianjin, 300387, P.R. China Full list of author information is available at the end of the article

\begin{abstract}
In this paper, our aim is to develop a compensated split-step $\theta(\operatorname{CSS} \theta)$ method for nonlinear jump-diffusion systems. First, we prove the convergence of the proposed method under a one-sided Lipschitz condition on the drift coefficient, and global Lipschitz condition on the diffusion and jump coefficients. Then we further show that the optimal strong convergence rate of $\operatorname{CSS} \theta$ can be recovered, if the drift coefficient satisfies a polynomial growth condition. At last, a nonlinear test equation is simulated to verify the results obtained from theory. The results show that the $\operatorname{CSS} \theta$ method is efficient for simulating the nonlinear jump-diffusion systems.
\end{abstract}

Keywords: jump-diffusion systems; nonlinear; compensated split-step $\theta$-method; convergence rate

\section{Introduction}

The aim of this paper is to study the strong convergence of the $\operatorname{CSS} \theta$ method for the following nonlinear jump-diffusion systems:

$$
\mathrm{d} X(t)=f\left(X\left(t^{-}\right)\right) \mathrm{d} t+g\left(X\left(t^{-}\right)\right) \mathrm{d} W(t)+h\left(X\left(t^{-}\right)\right) \mathrm{d} N(t)
$$

for $t>0$, with $X\left(0^{-}\right)=X_{0} \in \mathbb{R}^{n}$, where $X\left(t^{-}\right)$denotes $\lim _{s \rightarrow t^{-}} X(s), f: \mathbb{R}^{n} \rightarrow \mathbb{R}^{n}, g: \mathbb{R}^{n} \rightarrow$ $\mathbb{R}^{n \times m}, h: \mathbb{R}^{n} \rightarrow \mathbb{R}^{n}, W(t)$ is an $m$-dimensional Wiener process, and $N(t)$ is a scalar Poisson process with intensity $\lambda$.

Most of the studies concerned with numerical analysis for stochastic differential equations with jumps (SDEwJs) are based on the assumption of globally Lipschitz continuous coefficients, for example, [1-6]. However, they cannot be applied to many real-world models, such as financial models [7] and biology models [8], which violate the global Lipschitz assumptions. Hence, the development of numerical methods for SDEwJs under a nonglobally Lipschitz condition has become a focus point.

Firstly, we review some achievements of the numerical analysis for highly nonlinear SDEs. Here, we highlight work by Higham et al. [9], Hutzenthaler et al. [10], Szpruch and Mao [11], Mao and Szpruch [12], Huang [13], Zong et al. [14, 15].

However, the development of numerical methods for nonlinear jump-diffusion systems with non-globally Lipschitz continuous coefficients is not as fast as nonlinear SDEs. There 
are only few results on the numerical methods for nonlinear SDEwJs. For example, Higham and Kloeden proved the strong convergence and its order of the split-step backward Euler (SSBE) method and compensated split-step backward Euler (CSSBE) method for nonlinear jump-diffusion system in $[16,17]$. Huang applied the split-step $\theta$ (SS $\theta)$ method to SDEwJs, but he only studied the mean-square stability of the SS $\theta$ method for SDEwJs in [13]. To the best of our knowledge, there is no result about the strong convergence of the $\operatorname{CSS} \theta$ method for SDEwJs with non-globally Lipschitz continuous coefficients. The main difference of this paper from our previous work [5] is that we deal with SDEwJs with nonglobally Lipschitz condition on the drift coefficient $f$.

The outline of the paper is as follows. In Section 2, we introduce some notions and assumptions for SDEwJs. In Section 3, we construct the CSS $\theta$ method for nonlinear SDEwJs. In Section 4, the strong convergence of the numerical solutions produced by the CSS $\theta$ method is investigated. The convergence rate is studied in Section 5. Finally, a nonlinear numerical experiment is given to verify the convergence and efficiency of the proposed method.

\section{Conditions on the SDEwJs}

Let $(\Omega, \mathcal{F}, \mathbb{P})$ be a complete probability space with a filtration $\left\{\mathcal{F}_{t}\right\}_{t \geq 0}$, which satisfies the usual conditions, i.e., the filtration is continuous on the right and $\mathcal{F}_{0}$ contains all $\mathbb{P}$-null sets. Let $\langle\cdot, \cdot\rangle$ denote the Euclidean scalar product, and $|\cdot|$ denote both the Euclidean vector norm in $\mathbb{R}^{n}$ and the Frobenius matrix norm in $\mathbb{R}^{n \times m}$. For simplicity, we also denote $a \wedge b=\min \{a, b\}, a \vee b=\max \{a, b\}$.

Now, we give the following assumptions on the coefficients $f, g$ and $h$.

Assumption 2.1 The functions $f, g, h$ in (1.1) are $C^{1}$, there exist constants $K, L_{g}$ and $L_{h}>0$, such that the drift coefficient $f$ satisfies a one-sided Lipschitz condition,

$$
\langle x-y, f(x)-f(y)\rangle \leq K|x-y|^{2}, \quad \forall x, y \in \mathbb{R}^{n},
$$

and the diffusion and jump coefficients satisfy the global Lipschitz conditions,

$$
\begin{aligned}
& |g(x)-g(y)|^{2} \leq L_{g}|x-y|^{2}, \quad \forall x, y \in \mathbb{R}^{n}, \\
& |h(x)-h(y)|^{2} \leq L_{h}|x-y|^{2}, \quad \forall x, y \in \mathbb{R}^{n} .
\end{aligned}
$$

We also assume that all moments of the initial solution are bounded, that is, for any $p \in$ $[1,+\infty)$ there exists a positive constant $C$, such that

$$
\mathbb{E}\left|Y_{0}\right|^{p} \leq C
$$

Lemma 2.1 Under Assumption 2.1, equation (1.1) has a unique cadlag solution on $[0,+\infty)$.

Proof See [16], and for a more relaxed conditions see [18].

From Assumption 2.1, we have the following estimates:

$$
\langle x, f(x)\rangle=\langle x, f(x)-f(0)+f(0)\rangle \leq\left(K+\frac{1}{2}\right)|x|^{2}+\frac{1}{2}|f(0)|^{2},
$$




$$
\begin{aligned}
& |g(x)|^{2}=|g(x)-g(0)+g(0)|^{2} \leq 2 L_{g}|x|^{2}+2|g(0)|^{2}, \\
& |h(x)|^{2}=|h(x)-h(0)+h(0)|^{2} \leq 2 L_{h}|x|^{2}+2|h(0)|^{2} .
\end{aligned}
$$

It follows that

$$
\left\langle x,\left.f(x)|\vee| g(x)\right|^{2} \vee|h(x)|^{2} \leq L\left(1+|x|^{2}\right)\right.
$$

where $L=\max \left\{\left(K+\frac{1}{2}\right), 2 L_{g}, 2 L_{h}, \frac{1}{2}|f(0)|^{2}, 2|g(0)|^{2}, 2|h(0)|^{2}\right\}$.

\section{The compensated split-step $\theta$-method}

First defining

$$
f_{\lambda}:=f(x)+\lambda h(x)
$$

we can rewrite the jump-diffusion system (1.1) in the following form:

$$
\mathrm{d} X(t)=f_{\lambda}\left(X\left(t^{-}\right)\right) \mathrm{d} t+g\left(X\left(t^{-}\right)\right) \mathrm{d} W(t)+h\left(X\left(t^{-}\right)\right) \mathrm{d} \tilde{N}(t)
$$

where

$$
\tilde{N}(t):=N(t)-\lambda t
$$

is the compensated Poisson process.

Note that $f_{\lambda}$ satisfies the one-sided Lipschitz condition with lager constant; that is,

$$
\begin{aligned}
\left\langle x-y, f_{\lambda}(x)-f_{\lambda}(y)\right\rangle & \leq\left(K+\lambda \sqrt{L_{h}}\right)|x-y|^{2} \\
& :=K_{\lambda}|x-y|^{2}, \quad \forall x, y \in \mathbb{R}^{n} .
\end{aligned}
$$

Then we can get

$$
\left\langle x,\left.f_{\lambda}(x)|\vee| g(x)\right|^{2} \vee|h(x)|^{2} \leq L_{\lambda}\left(1+|x|^{2}\right),\right.
$$

where $L_{\lambda}=\max \left\{\left(K+\lambda \sqrt{L_{h}}+\frac{1}{2}\right), 2 L_{g}, 2 L_{h}, \frac{1}{2}\left|f_{\lambda}(0)\right|^{2}, 2|g(0)|^{2}, 2|h(0)|^{2}\right\}$.

Now we define the $\operatorname{CSS} \theta$ method for the jump-diffusion system (1.1) by $Y_{0}=X(0)$ and

$$
\begin{aligned}
& Y_{n}^{*}=Y_{n}+\theta f_{\lambda}\left(Y_{n}^{*}\right) \Delta t, \\
& Y_{n+1}=Y_{n}+f_{\lambda}\left(Y_{n}^{*}\right) \Delta t+g\left(Y_{n}^{*}\right) \Delta W_{n}+h\left(Y_{n}^{*}\right) \Delta \tilde{N}_{n},
\end{aligned}
$$

where $\theta \in[0,1], \Delta t>0, Y_{n}$ is the numerical approximation of $X\left(t_{n}\right)$ with $t_{n}=n \cdot \Delta t$. Moreover, $\Delta W_{n}:=W\left(t_{n+1}\right)-W\left(t_{n}\right), \Delta \tilde{N}_{n}:=\tilde{N}\left(t_{n+1}\right)-\tilde{N}\left(t_{n}\right)$ are independent increments of the Wiener process and Poisson process, respectively.

If we have $\theta=1$, the $\operatorname{CSS} \theta$ method becomes the CSSBE method in [16].

Since the $\operatorname{CSS} \theta$ method is an implicit scheme, we need to make sure that equation (3.4) has a unique solution $Y_{n}^{*}$ given $Y_{n}$. 
In fact, under the one-sided Lipschitz condition (3.2) with $\theta \Delta t K_{\lambda}<1$, equation (3.4) admits a unique solution (see [12]). Meanwhile, if $K_{\lambda}<0$, then $\theta \Delta t K_{\lambda}<1$ holds for any $\Delta t>0$. Hence, we define

$$
\Delta= \begin{cases}\infty, & \text { if } K_{\lambda}<0, \text { or } \theta=0 \\ \frac{1}{\theta K_{\lambda}}, & \text { if } K_{\lambda}>0, \theta \in(0,1]\end{cases}
$$

From now on we always assume that $\Delta t \leq \Delta$.

\section{Strong convergence on finite time interval $[0, T]$}

First, for $t \in\left[t_{n}, t_{n+1}\right)$, we define the step function:

$$
Y(t)=\sum_{n=0}^{N_{T}-1} Y_{n}^{*} I_{[n \Delta,(n+1) \Delta)}(t)
$$

where $N_{T}$ is the largest number such that $N_{T} \Delta t \leq T$, and $I_{A}$ is the indicator function for the set $\mathrm{A}$, i.e.,

$$
I_{A}(x)= \begin{cases}1, & x \in A, \\ 0, & x \notin A .\end{cases}
$$

Then we define the continuous-time approximations

$$
\begin{aligned}
\bar{Y}(t)= & Y_{n}+f_{\lambda}\left(Y_{n}^{*}\right)\left(t-t_{n}\right)+g\left(Y_{n}^{*}\right)\left(W(t)-W\left(t_{n}\right)\right) \\
& +h\left(Y_{n}^{*}\right)\left(\tilde{N}(t)-\tilde{N}\left(t_{n}\right)\right), \quad t \in\left[t_{n}, t_{n+1}\right) .
\end{aligned}
$$

Thus we can rewrite (4.2) in integral form:

$$
\begin{aligned}
\bar{Y}(t)= & Y_{0}+\int_{0}^{t} f_{\lambda}\left(Y\left(s^{-}\right)\right) \mathrm{d} s+\int_{0}^{t} g\left(Y\left(s^{-}\right)\right) \mathrm{d} W(s) \\
& +\int_{0}^{t} h\left(Y\left(s^{-}\right)\right) \mathrm{d} \tilde{N}(s) .
\end{aligned}
$$

It is easy to verify that $\bar{Y}\left(t_{n}\right)=Y_{n}$, that is, $\bar{Y}(t)$ is a continuous-time extension of the discrete approximation $\left\{Y_{n}\right\}$.

Now we will prove the strong convergence of the $\operatorname{CSS} \theta$ method. The main technique of the following proof is based on the fundamental papers $[9,13,16]$, we refer to them for a fuller description of some of the technical details.

The following two lemmas show the $p$ th moment properties of the true solutions and numerical solutions.

Lemma 4.1 Let Assumption 2.1 hold, and $0<\theta \leq 1, p \geq 1,0<\Delta t<\min \left\{1, \frac{1}{2 \theta L_{\lambda}}\right\}$, then there exists a positive constant $A$ independent of $N_{T}$ such that

$$
\mathbb{E}\left(\sup _{0 \leq n \Delta t \leq T}\left|Y_{n}\right|^{2 p}\right) \vee \mathbb{E}\left(\sup _{0 \leq n \Delta t \leq T}\left|Y_{n}^{*}\right|^{2 p}\right)<A,
$$

where $Y_{n}^{*}$ and $Y_{n}$ are produced by (3.4) and (3.5). 
Proof In the following we assume that $M$ is a positive integer such that $n \Delta t \leq M \Delta t \leq T$. Squaring both sides of (3.4), we find

$$
\begin{aligned}
\left|Y_{n}^{*}\right|^{2} & =\left|Y_{n}+\theta \Delta t f_{\lambda}\left(Y_{n}^{*}\right)\right|^{2} \\
& =\left|Y_{n}\right|^{2}+\theta^{2} \Delta t^{2}\left|f_{\lambda}\left(Y_{n}^{*}\right)\right|^{2}+2 \theta \Delta t\left\langle Y_{n}, f_{\lambda}\left(Y_{n}^{*}\right)\right\rangle
\end{aligned}
$$

and

$$
\left\langle Y_{n}, f_{\lambda}\left(Y_{n}^{*}\right)\right\rangle=\left\langle Y_{n}^{*}, f_{\lambda}\left(Y_{n}^{*}\right)\right\rangle-\theta \Delta t\left\langle f_{\lambda}\left(Y_{n}^{*}\right), f_{\lambda}\left(Y_{n}^{*}\right)\right\rangle .
$$

Substituting (4.5) into (4.4), we have

$$
\begin{aligned}
\left|Y_{n}^{*}\right|^{2} & =\left|Y_{n}\right|^{2}-\theta^{2} \Delta t^{2}\left\langle f_{\lambda}\left(Y_{n}^{*}\right), f_{\lambda}\left(Y_{n}^{*}\right)\right\rangle+2 \theta \Delta t\left\langle Y_{n}^{*}, f_{\lambda}\left(Y_{n}^{*}\right)\right\rangle \\
& \leq\left|Y_{n}\right|^{2}+2 \theta \Delta t\left\langle Y_{n}^{*}, f_{\lambda}\left(Y_{n}^{*}\right)\right\rangle \\
& \leq\left|Y_{n}\right|^{2}+2 \theta \Delta t L_{\lambda}\left(1+\left|Y_{n}^{*}\right|^{2}\right)
\end{aligned}
$$

which gives

$$
\begin{aligned}
\left|Y_{n}^{*}\right|^{2} & \leq \frac{1}{1-2 \theta \Delta t L_{\lambda}}\left|Y_{n}\right|^{2}+\frac{2 \theta \Delta t L_{\lambda}}{1-2 \theta \Delta t L_{\lambda}} \\
& =\left|Y_{n}\right|^{2}+\frac{2 \theta \Delta t L_{\lambda}}{1-2 \theta \Delta t L_{\lambda}}\left|Y_{n}\right|^{2}+\frac{2 \theta \Delta t L_{\lambda}}{1-2 \theta \Delta t L_{\lambda}} \\
& =\left|Y_{n}\right|^{2}+\alpha\left|Y_{n}\right|^{2}+\alpha \\
& =\beta\left|Y_{n}\right|^{2}+\alpha,
\end{aligned}
$$

where $\alpha=\frac{2 \theta \Delta t L_{\lambda}}{1-2 \theta \Delta t L_{\lambda}}, \beta=1+\alpha$. By (3.5) we have

$$
\begin{aligned}
\left|Y_{n+1}\right|^{2}= & \left|Y_{n}+f_{\lambda}\left(Y_{n}^{*}\right) \Delta t+g\left(Y_{n}^{*}\right) \Delta W_{n}+h\left(Y_{n}^{*}\right) \Delta \tilde{N}_{n}\right|^{2} \\
= & \left|Y_{n}\right|^{2}+\left|f_{\lambda}\left(Y_{n}^{*}\right) \Delta t\right|^{2}+\left|g\left(Y_{n}^{*}\right) \Delta W_{n}\right|^{2}+\left|h\left(Y_{n}^{*}\right) \Delta \tilde{N}_{n}\right|^{2} \\
& +2\left\langle Y_{n}, f_{\lambda}\left(Y_{n}^{*}\right) \Delta t\right\rangle+2\left\langle Y_{n}, g\left(Y_{n}^{*}\right) \Delta W_{n}\right\rangle+2\left\langle Y_{n}, h\left(Y_{n}^{*}\right) \Delta \tilde{N}_{n}\right\rangle \\
& +2\left\langle f_{\lambda}\left(Y_{n}^{*}\right) \Delta t, g\left(Y_{n}^{*}\right) \Delta W_{n}\right\rangle \\
& +2\left\langle f_{\lambda}\left(Y_{n}^{*}\right) \Delta t, h\left(Y_{n}^{*}\right) \Delta \tilde{N}_{n}\right\rangle \\
& +2\left\langle g\left(Y_{n}^{*}\right) \Delta W_{n}, h\left(Y_{n}^{*}\right) \Delta \tilde{N}_{n}\right\rangle .
\end{aligned}
$$

Then by (3.3), (3.4) and (4.5), we get

$$
\begin{aligned}
\left|Y_{n+1}\right|^{2} \leq & \left|Y_{n}\right|^{2}+\left|g\left(Y_{n}^{*}\right) \Delta W_{n}\right|^{2}+\left|h\left(Y_{n}^{*}\right) \Delta \tilde{N}_{n}\right|^{2}+2\left\langle Y_{n}^{*}, f_{\lambda}\left(Y_{n}^{*}\right) \Delta t\right\rangle \\
& +2\left\langle Y_{n}, g\left(Y_{n}^{*}\right) \Delta W_{n}\right\rangle+2\left\langle Y_{n}, h\left(Y_{n}^{*}\right) \Delta \tilde{N}_{n}\right\rangle \\
& +2\left\langle\frac{Y_{n}^{*}-Y_{n}}{\theta}, g\left(Y_{n}^{*}\right) \Delta W_{n}\right\rangle \\
& +2\left\langle\frac{Y_{n}^{*}-Y_{n}}{\theta}, h\left(Y_{n}^{*}\right) \Delta \tilde{N}_{n}\right\rangle \\
& +2\left\langle g\left(Y_{n}^{*}\right) \Delta W_{n}, h\left(Y_{n}^{*}\right) \Delta \tilde{N}_{n}\right\rangle
\end{aligned}
$$




$$
\begin{aligned}
\leq & \left|Y_{n}\right|^{2}+\left|g\left(Y_{n}^{*}\right) \Delta W_{n}\right|^{2}+\left|h\left(Y_{n}^{*}\right) \Delta \tilde{N}_{n}\right|^{2}+2 L_{\lambda} \Delta t\left(1+\left|Y_{n}^{*}\right|^{2}\right) \\
& +2\left(1-\frac{1}{\theta}\right)\left\langle Y_{n}, g\left(Y_{n}^{*}\right) \Delta W_{n}\right\rangle \\
& +2\left(1-\frac{1}{\theta}\right)\left\langle Y_{n}, h\left(Y_{n}^{*}\right) \Delta \tilde{N}_{n}\right\rangle \\
& +\frac{2}{\theta}\left\langle Y_{n}^{*}, g\left(Y_{n}^{*}\right) \Delta W_{n}\right\rangle+\frac{2}{\theta}\left\langle Y_{n}^{*}, h\left(Y_{n}^{*}\right) \Delta \tilde{N}_{n}\right\rangle \\
& +2\left\langle g\left(Y_{n}^{*}\right) \Delta W_{n}, h\left(Y_{n}^{*}\right) \Delta \tilde{N}_{n}\right\rangle .
\end{aligned}
$$

Hence from (4.7) we have

$$
\begin{aligned}
\left|Y_{n+1}\right|^{2} \leq & \left|Y_{n}\right|^{2}+2 \beta L_{\lambda} \Delta t\left|Y_{n}\right|^{2}+2(\alpha+1) L_{\lambda} \Delta t \\
& +\left|g\left(Y_{n}^{*}\right) \Delta W_{n}\right|^{2}+\left|h\left(Y_{n}^{*}\right) \Delta \tilde{N}_{n}\right|^{2} \\
& +2\left(1-\frac{1}{\theta}\right)\left\langle Y_{n}, g\left(Y_{n}^{*}\right) \Delta W_{n}\right\rangle \\
& +2\left(1-\frac{1}{\theta}\right)\left\langle Y_{n}, h\left(Y_{n}^{*}\right) \Delta \tilde{N}_{n}\right\rangle \\
& +\frac{2}{\theta}\left\langle Y_{n}^{*}, g\left(Y_{n}^{*}\right) \Delta W_{n}\right\rangle+\frac{2}{\theta}\left\langle Y_{n}^{*}, h\left(Y_{n}^{*}\right) \Delta \tilde{N}_{n}\right\rangle \\
& +2\left\langle g\left(Y_{n}^{*}\right) \Delta W_{n}, h\left(Y_{n}^{*}\right) \Delta \tilde{N}_{n}\right\rangle .
\end{aligned}
$$

By the recursive calculation, we can get

$$
\begin{aligned}
\left|Y_{n}\right|^{2} \leq & \left|Y_{0}\right|^{2}+2 \beta L_{\lambda} \Delta t \sum_{j=0}^{n-1}\left|Y_{j}\right|^{2}+2(\alpha+1) L_{\lambda} T \\
& +\sum_{j=0}^{n-1}\left|g\left(Y_{j}^{*}\right) \Delta W_{j}\right|^{2}+\sum_{j=0}^{n-1}\left|h\left(Y_{j}^{*}\right) \Delta \tilde{N}_{j}\right|^{2} \\
& +2\left(1-\frac{1}{\theta}\right) \sum_{j=0}^{n-1}\left\langle Y_{j}, g\left(Y_{j}^{*}\right) \Delta W_{j}\right\rangle \\
& +2\left(1-\frac{1}{\theta}\right) \sum_{j=0}^{n-1}\left\langle Y_{j}, h\left(Y_{j}^{*}\right) \Delta \tilde{N}_{j}\right\rangle \\
& +\frac{2}{\theta} \sum_{j=0}^{n-1}\left\langle Y_{j}^{*}, g\left(Y_{j}^{*}\right) \Delta W_{j}\right\rangle+\frac{2}{\theta} \sum_{j=0}^{n-1}\left\langle Y_{j}^{*}, h\left(Y_{j}^{*}\right) \Delta \tilde{N}_{j}\right\rangle \\
& +2 \sum_{j=0}^{n-1}\left\langle g\left(Y_{j}^{*}\right) \Delta W_{j}, h\left(Y_{j}^{*}\right) \Delta \tilde{N}_{j}\right\rangle .
\end{aligned}
$$

Raising both sides to the power $p$, we can obtain

$$
\begin{aligned}
\left|Y_{n}\right|^{2 p} \leq & 10^{p-1}\left\{\left|Y_{0}\right|^{2 p}+n^{p-1}\left(2 \beta L_{\lambda} \Delta t\right)^{p} \sum_{j=0}^{n-1}\left|Y_{j}\right|^{2 p}+\left(2(\alpha+1) L_{\lambda} T\right)^{p}\right. \\
& +n^{p-1} \sum_{j=0}^{n-1}\left|g\left(Y_{j}^{*}\right) \Delta W_{j}\right|^{2 p}+n^{p-1} \sum_{j=0}^{n-1}\left|h\left(Y_{j}^{*}\right) \Delta \tilde{N}_{j}\right|^{2 p}
\end{aligned}
$$




$$
\begin{aligned}
& +2^{p}\left(\frac{1}{\theta}-1\right)^{p}\left|\sum_{j=0}^{n-1}\left\langle Y_{j}, g\left(Y_{j}^{*}\right) \Delta W_{j}\right\rangle\right|^{p} \\
& +2^{p}\left(\frac{1}{\theta}-1\right)^{p}\left|\sum_{j=0}^{n-1}\left\langle Y_{j}, h\left(Y_{j}^{*}\right) \Delta \tilde{N}_{j}\right\rangle\right|^{p} \\
& +2^{p}\left(\frac{2}{\theta}\right)^{p}\left|\sum_{j=0}^{n-1}\left\langle Y_{j}^{*}, g\left(Y_{j}^{*}\right) \Delta W_{j}\right\rangle\right|^{p} \\
& +2^{p}\left(\frac{2}{\theta}\right)^{p}\left|\sum_{j=0}^{n-1}\left\langle Y_{j}^{*}, h\left(Y_{j}^{*}\right) \Delta \tilde{N}_{j}\right\rangle\right|^{p} \\
& \left.+2^{p}\left|\sum_{j=0}^{n-1}\left\langle g\left(Y_{j}^{*}\right) \Delta W_{j}, h\left(Y_{j}^{*}\right) \Delta \tilde{N}_{j}\right\rangle\right|^{p}\right\} .
\end{aligned}
$$

Notice that

$$
\mathbb{E} \sup _{0 \leq n \leq M}\left[\sum_{j=0}^{n-1}\left|Y_{j}\right|^{2 p}\right]=\sum_{j=0}^{M-1} \mathbb{E}\left|Y_{j}\right|^{2 p} .
$$

Thus, for $0 \leq M \leq N_{T}$, we obtain

$$
\begin{aligned}
\mathbb{E} \sup _{0 \leq n \leq M}\left|Y_{n}\right|^{2 p} \leq & 10^{p-1}\left\{\left|Y_{0}\right|^{2 p}+n^{p-1}\left(2 \beta L_{\lambda} \Delta t\right)^{p} \sum_{j=0}^{M-1} \mathbb{E}\left|Y_{j}\right|^{2 p}+\left(2(\alpha+1) L_{\lambda} T\right)^{p}\right. \\
& +n^{p-1} \mathbb{E} \sup _{0 \leq n \leq M} \sum_{j=0}^{n-1}\left|g\left(Y_{j}^{*}\right) \Delta W_{j}\right|^{2 p} \\
& +n^{p-1} \mathbb{E} \sup _{0 \leq n \leq M} \sum_{j=0}^{n-1}\left|h\left(Y_{j}^{*}\right) \Delta \tilde{N}_{j}\right|^{2 p} \\
& +2^{p}\left(\frac{1}{\theta}-1\right)^{p} \mathbb{E} \sup _{0 \leq n \leq M}\left|\sum_{j=0}^{n-1}\left\langle Y_{j}, g\left(Y_{j}^{*}\right) \Delta W_{j}\right\rangle\right|^{p} \\
& +2^{p}\left(\frac{1}{\theta}-1\right)^{p} \mathbb{E} \sup _{0 \leq n \leq M} \mid \sum_{j=0}^{n-1}\left\langle Y_{j}, h\left(Y_{j}^{*}\right) \Delta \tilde{N}_{j}||^{p}\right. \\
& +2^{p}\left(\frac{2}{\theta}\right)^{p} \mathbb{E} \mid \sum_{j=0}^{n-1}\left\langle Y_{j}^{*}, g\left(Y_{j}^{*}\right) \Delta W_{j}||^{p}\right. \\
& +2^{p}\left(\frac{2}{\theta}\right)^{p} \mathbb{E}\left|\sum_{j=0}^{n-1}\left\langle Y_{j}^{*}, h\left(Y_{j}^{*}\right) \Delta \tilde{N}_{j}\right\rangle\right|^{p} \\
& \left.+2^{p} \mathbb{E} \sup _{0 \leq n \leq M}\left|\sum_{j=0}^{n-1}\left\langle g\left(Y_{j}^{*}\right) \Delta W_{j}, h\left(Y_{j}^{*}\right) \Delta \tilde{N}_{j}\right\rangle\right|^{p}\right\} .
\end{aligned}
$$

To bound the fourth term on the right side of (4.14), we note that $Y_{n}^{*} \in \mathcal{F}_{t_{n}}, \Delta W_{n}$ is independent of $\mathcal{F}_{t_{n}}$ and $\mathbb{E}\left|\Delta W_{j}\right|^{2 p} \leq c_{p} \Delta t^{p}$, where $c_{p}$ is a constant. Meanwhile, letting 
$C=C\left(p, T, L_{\lambda}, \theta\right)$ be a constant that may change from line to line,

$$
\begin{aligned}
n^{p-1} \mathbb{E} \sup _{0 \leq n \leq M} \sum_{j=0}^{n-1}\left|g\left(Y_{j}^{*}\right) \Delta W_{j}\right|^{2 p} & =n^{p-1} \mathbb{E} \sum_{j=0}^{M-1}\left|g\left(Y_{j}^{*}\right) \Delta W_{j}\right|^{2 p} \\
& \leq n^{p-1} \sum_{j=0}^{M-1} \mathbb{E}\left|g\left(Y_{j}^{*}\right)\right|^{2 p} \mathbb{E}\left|\Delta W_{j}\right|^{2 p} \\
& \leq n^{p-1} c_{p} \Delta t^{p} L_{\lambda}^{p} \sum_{j=0}^{M-1} \mathbb{E}\left[1+\left|Y_{j}^{*}\right|^{2}\right]^{p} \\
& \leq T^{p-1} c_{p} \Delta t L_{\lambda}^{p} \sum_{j=0}^{M-1} \mathbb{E}\left[1+\beta\left|Y_{j}\right|^{2}+\alpha\right]^{p} \\
& \leq 2^{p-1} T^{p-1} c_{p} \Delta t L_{\lambda}^{p} \sum_{j=0}^{M-1} \mathbb{E}\left[\beta^{p}+\beta^{p}\left|Y_{j}\right|^{2 p}\right] \\
& \leq(2 T)^{p-1} c_{p} \Delta t L_{\lambda}^{p} \beta^{p}\left(M+\sum_{j=0}^{M-1} \mathbb{E}\left|Y_{j}\right|^{2 p}\right) \\
& \leq C+C \Delta t \sum_{j=0}^{M-1} \mathbb{E}\left|Y_{j}\right|^{2 p} .
\end{aligned}
$$

Using a similar approach to the fifth term and noticing that $\mathbb{E}\left|\Delta \widetilde{N}_{j}\right|^{2 p} \leq c_{p} \Delta t^{p}$, we have

$$
n^{p-1} \mathbb{E} \sum_{j=0}^{M-1}\left|h\left(Y_{j}^{*}\right) \Delta \tilde{N}_{j}\right|^{2 p} \leq C+C \Delta t \sum_{j=0}^{M-1} \mathbb{E}\left|Y_{j}\right|^{2 p} .
$$

Now we bound the sixth term in (4.14), using the Burkholder-Davis-Gundy inequality

$$
\begin{aligned}
\mathbb{E} \sup _{0 \leq n \leq M} \mid \sum_{j=0}^{n-1}\left\langle Y_{j}, g\left(Y_{j}^{*}\right) \Delta W_{j}||^{p}\right. & \leq C \mathbb{E}\left[\sum_{j=0}^{M-1}\left|Y_{j}\right|^{2}\left|g\left(Y_{j}^{*}\right)\right|^{2} \Delta t\right]^{p / 2} \\
& \leq C \Delta t^{p / 2} M^{p / 2-1} L_{\lambda}^{p} \mathbb{E} \sum_{j=0}^{M-1}\left|Y_{j}\right|^{p}\left(1+\left|Y_{j}^{*}\right|^{2}\right)^{p / 2} \\
& \leq C T^{p / 2-1} \Delta t \mathbb{E} \sum_{j=0}^{M-1} \frac{\left|Y_{j}\right|^{2 p}+\left(1+\left|Y_{j}^{*}\right|^{2}\right)^{p}}{2} \\
& \leq C \Delta t \mathbb{E} \sum_{j=0}^{M-1}\left[\left|Y_{j}\right|^{2 p}+2^{p-1}+2^{p-1}\left|Y_{j}^{*}\right|^{2 p}\right] \\
& \leq C \Delta t \mathbb{E} \sum_{j=0}^{M-1}\left[\left|Y_{j}\right|^{2 p}+2^{p-1}\left(\beta\left|Y_{j}\right|^{2}+\alpha\right)^{p}+2^{p-1}\right] \\
& \leq C+C \Delta t \sum_{j=0}^{M-1} \mathbb{E}\left|Y_{j}\right|^{2 p} .
\end{aligned}
$$


Similar to the sixth term, we can bound the seventh term

$$
\mathbb{E} \sup _{0 \leq n \leq M}\left|\sum_{j=0}^{n-1}\left\langle Y_{j}, h\left(Y_{j}^{*}\right) \Delta \tilde{N}_{j}\right)\right|^{p} \leq C+C \Delta t \sum_{j=0}^{M-1} \mathbb{E}\left|Y_{j}\right|^{2 p} .
$$

Also similar to the sixth term, we can bound the eighth term in (4.14),

$$
\begin{aligned}
\mathbb{E} \sup _{0 \leq n \leq M}\left|\sum_{j=0}^{n-1}\left\langle Y_{j}^{*}, g\left(Y_{j}^{*}\right) \Delta W_{j}\right\rangle\right|^{p} & \leq C \mathbb{E}\left[\sum_{j=0}^{M-1}\left|Y_{j}^{*}\right|^{2}\left|g\left(Y_{j}^{*}\right)\right|^{2} \Delta t\right]^{p / 2} \\
& \leq C \Delta t^{p / 2} M^{p / 2-1} L_{\lambda}^{p} \mathbb{E} \sum_{j=0}^{M-1}\left|Y_{j}^{*}\right|^{p}\left(1+\left|Y_{j}^{*}\right|^{2}\right)^{p / 2} \\
& \leq C T^{p / 2-1} \Delta t \mathbb{E} \sum_{j=0}^{M-1} \frac{\left|Y_{j}^{*}\right|^{2 p}+\left(1+\left|Y_{j}^{*}\right|^{2}\right)^{p}}{2} \\
& \leq C \Delta t \mathbb{E} \sum_{j=0}^{M-1}\left[\left|Y_{j}^{*}\right|^{2 p}+2^{p-1}+2^{p-1}\left|Y_{j}^{*}\right|^{2 p}\right] \\
& \leq C \Delta t \mathbb{E} \sum_{j=0}^{M-1}\left[\left(1+2^{p-1}\right)\left(\beta\left|Y_{j}\right|^{2}+\alpha\right)^{p}+2^{p-1}\right] \\
& \leq C+C \Delta t \sum_{j=0}^{M-1} \mathbb{E}\left|Y_{j}\right|^{2 p}
\end{aligned}
$$

and the ninth term,

$$
\mathbb{E} \sup _{0 \leq n \leq M}\left|\sum_{j=0}^{n-1}\left\langle Y_{j}^{*}, h\left(Y_{j}^{*}\right) \Delta \widetilde{N}_{j}\right)\right|^{p} \leq C+C \Delta t \sum_{j=0}^{M-1} \mathbb{E}\left|Y_{j}\right|^{2 p} .
$$

Finally we bound the tenth term in (4.14), by (4.15)-(4.16); we have

$$
\begin{aligned}
\left.\mathbb{E} \sup _{0 \leq n \leq M}\left|\sum_{j=0}^{n-1}\right| g\left(Y_{j}^{*}\right) \Delta W_{j}, h\left(Y_{j}^{*}\right) \Delta \tilde{N}_{j}\right)\left.\right|^{p} \\
\leq 2^{-p} \mathbb{E} \sup _{0 \leq n \leq M}\left|\sum_{j=0}^{n-1}\left(\left|g\left(Y_{j}^{*}\right) \Delta W_{j}\right|^{2}+\left|h\left(Y_{j}^{*}\right) \Delta \tilde{N}_{j}\right|^{2}\right)\right|^{p} \\
\leq 2^{-p} M^{p-1} \mathbb{E} \sup _{0 \leq n \leq M} \sum_{j=0}^{n-1}\left(\left|g\left(Y_{j}^{*}\right) \Delta W_{j}\right|^{2}+\left|h\left(Y_{j}^{*}\right) \Delta \tilde{N}_{j}\right|^{2}\right)^{p} \\
\leq 2^{-1} M^{p-1} \mathbb{E} \sup _{0 \leq n \leq M} \sum_{j=0}^{n-1}\left(\left|g\left(Y_{j}^{*}\right) \Delta W_{j}\right|^{2 p}+\left|h\left(Y_{j}^{*}\right) \Delta \tilde{N}_{j}\right|^{2 p}\right) \\
\leq C+C \Delta t \sum_{j=0}^{M-1} \mathbb{E}\left|Y_{j}\right|^{2 p} .
\end{aligned}
$$


Combining (4.15)-(4.21) into (4.14), we obtain

$$
\begin{aligned}
\mathbb{E} \sup _{0 \leq n \leq M}\left|Y_{n}\right|^{2 p} & \leq C+C \Delta t \sum_{j=0}^{M-1} \mathbb{E}\left|Y_{j}\right|^{2 p} \\
& \leq C+C \Delta t \sum_{j=0}^{M-1} \mathbb{E} \sup _{0 \leq n \leq j}\left|Y_{n}\right|^{2 p} .
\end{aligned}
$$

Using the discrete-type Gronwall inequality and noting that $M \Delta t \leq T$, we obtain

$$
\mathbb{E} \sup _{0 \leq n \leq M}\left|Y_{n}\right|^{2 p} \leq C e^{C \Delta t M} \leq C e^{C T}
$$

By (4.7), we find that $\mathbb{E} \sup _{0 \leq n \leq M}\left|Y_{n}^{*}\right|^{2 p}$ is also bounded.

Lemma 4.2 Let Assumption 2.1 hold, and $0<\theta \leq 1, p \geq 1,0<\Delta t<\min \left\{1, \frac{1}{2 \theta L_{\lambda}}\right\}$, then the exact solution of (3.1) and the continuous-time extension (4.3) satisfy

$$
\mathbb{E}\left(\sup _{0 \leq t \leq T}|X(t)|^{2 p}\right) \vee \mathbb{E}\left(\sup _{0 \leq t \leq T}|\bar{Y}(t)|^{2 p}\right)<A_{1}
$$

where $A_{1}$ is a positive constant independent of $N_{T}$.

Proof From Lemma 1 in [16], we can see that $\mathbb{E}\left(\sup _{0 \leq t \leq T}|X(t)|^{2 p}\right)$ is bounded. Now we prove that $\mathbb{E}\left(\sup _{0 \leq t \leq T}|\bar{Y}(t)|^{2 p}\right)$ is bounded.

From (4.2), we obtain

$$
\begin{aligned}
\bar{Y}(t)= & Y_{n}+f_{\lambda}\left(Y_{n}^{*}\right)\left(t-t_{n}\right)+g\left(Y_{n}^{*}\right)\left(W(t)-W\left(t_{n}\right)\right) \\
& +h\left(Y_{n}^{*}\right)\left(\tilde{N}(t)-\tilde{N}\left(t_{n}\right)\right), \quad t \in\left[t_{n}, t_{n+1}\right) .
\end{aligned}
$$

Let $s \in[0, \Delta t)$, we have

$$
\bar{Y}\left(t_{n}+s\right)=Y_{n}+f_{\lambda}\left(Y_{n}^{*}\right) s+g\left(Y_{n}^{*}\right) \Delta W_{n}(s)+h\left(Y_{n}^{*}\right) \Delta \tilde{N}_{n}(s)
$$

where

$$
\begin{aligned}
& \Delta W_{n}(s)=W\left(t_{n}+s\right)-W\left(t_{n}\right), \\
& \Delta \widetilde{N}_{n}(s)=\widetilde{N}\left(t_{n}+s\right)-\tilde{N}\left(t_{n}\right) .
\end{aligned}
$$

However, $Y_{n}^{*}=Y_{n}+\theta \Delta t f_{\lambda}\left(Y_{n}^{*}\right)$ and so, for $a=s / \Delta t$, we can rewrite equation (4.25) in the following form:

$$
\bar{Y}\left(t_{n}+s\right)=\frac{a}{\theta} Y_{n}^{*}+\left(1-\frac{a}{\theta}\right) Y_{n}+g\left(Y_{n}^{*}\right) \Delta W_{n}(s)+h\left(Y_{n}^{*}\right) \Delta \widetilde{N}_{n}(s)
$$

By (4.7), we have

$$
\left|\bar{Y}\left(t_{n}+s\right)\right|^{2} \leq C\left[1+\left|Y_{n}\right|^{2}+\left|g\left(Y_{n}^{*}\right) \Delta W_{n}(s)\right|^{2}+\left|h\left(Y_{n}^{*}\right) \Delta \tilde{N}_{n}(s)\right|^{2}\right]
$$


Thus

$$
\begin{aligned}
& \sup _{0 \leq t \leq T}|\bar{Y}(t)|^{2 p} \\
& \leq \sup _{0 \leq n \Delta t \leq T} \sup _{0 \leq s \leq \Delta t}\left|\bar{Y}\left(t_{n}+s\right)\right|^{2 p} \\
& \leq \sup _{0 \leq n \Delta t \leq T} \sup _{0 \leq s \leq \Delta t} C\left[1+\left|Y_{n}\right|^{2 p}+\left|g\left(Y_{n}^{*}\right) \Delta W_{n}(s)\right|^{2 p}+\left|h\left(Y_{n}^{*}\right) \Delta \tilde{N}_{n}(s)\right|^{2 p}\right] \\
& \leq C\left[1+\sup _{0 \leq n \Delta t \leq T}\left|Y_{n}\right|^{2 p}+\sup _{0 \leq s \leq \Delta t} \sum_{j=0}^{N_{T}}\left|g\left(Y_{j}^{*}\right) \Delta W_{j}(s)\right|^{2 p}\right. \\
& \left.\quad+\sup _{0 \leq s \leq \Delta t} \sum_{j=0}^{N_{T}}\left|h\left(Y_{j}^{*}\right) \Delta \tilde{N}_{j}(s)\right|^{2 p}\right] .
\end{aligned}
$$

Now using Doob's martingale inequality, (3.3) and Lemma 4.1, we have

$$
\begin{aligned}
\mathbb{E} \sup _{0 \leq s \leq \Delta t}\left|g\left(Y_{j}^{*}\right) \Delta W_{j}(s)\right|^{2 p} & \leq C \mathbb{E}\left|g\left(Y_{j}^{*}\right) \Delta W_{j}(\Delta t)\right|^{2 p} \\
& \leq C \mathbb{E}\left|g\left(Y_{j}^{*}\right)\right|^{2 p} \mathbb{E}\left|\Delta W_{j}(\Delta t)\right|^{2 p} \\
& \leq C\left(1+\mathbb{E}\left|Y_{j}^{*}\right|^{2 p}\right) \Delta t^{p} \\
& \leq C \Delta t .
\end{aligned}
$$

Since the $\Delta \widetilde{N}_{j}(s)$ is also a martingale, by a similar method, we get

$$
\mathbb{E} \sup _{0 \leq s \leq \Delta t}\left|h\left(Y_{j}^{*}\right) \Delta \tilde{N}_{j}(s)\right|^{2 p} \leq C \Delta t .
$$

Then by (4.28), (4.29) and Lemma 4.1, combining $N_{T} \Delta t \leq T$, we have

$$
\mathbb{E}\left(\sup _{0 \leq t \leq T}|\bar{Y}(t)|^{2 p}\right) \leq A_{1}
$$

Then we get the desired results.

Now we use the above lemmas to prove a strong convergence result.

Remark 4.1 Since $f(x) \in C^{1}$, i.e. $f^{\prime}(x)$ is continuous, $\left|f^{\prime}(x)\right|$ is bounded locally. Then by the mean value theorem, there exists a positive constant $L_{R}$ for each $R>0$, such that

$$
|f(x)-f(y)|=\left|f^{\prime}(z)\right||x-y| \leq L_{R}|x-y|
$$

for all $x, y, z \in \mathbb{R}^{n}$ with $|x| \vee|y| \leq R$.

We note that the function $f_{\lambda}$ in (3.1) automatically inherits this condition, with a larger $L_{R}$.

Theorem 4.3 Under Assumption 2.1, let $0<\theta \leq 1,0<\Delta t<\min \left\{1, \frac{1}{2 \theta L_{\lambda}}\right\}$, the continuoustime approximate solution $\bar{Y}(t)$ defined by (4.3) will converge to the true solution $X(t)$ of 
(3.1) in the mean-square sense, i.e.

$$
\lim _{\Delta t \rightarrow 0} \mathbb{E} \sup _{0 \leq t \leq T}|\bar{Y}(t)-X(t)|^{2}=0
$$

Proof First, we define

$$
\tau_{d}:=\inf \{t \geq 0,|X(t)| \geq d\}, \quad \sigma_{d}:=\inf \{t \geq 0,|\bar{Y}(t)| \geq d\}, \quad v_{d}=\tau_{d} \wedge \sigma_{d}
$$

and let

$$
e(t)=\bar{Y}(t)-X(t)
$$

Recall the Young inequality: for $\frac{1}{p}+\frac{1}{q}=1(p, q>0)$, we have

$$
a b=a \delta^{\frac{1}{p}} \frac{b}{\delta^{\frac{1}{p}}} \leq \frac{\left(a \delta^{\frac{1}{p}}\right)^{p}}{p}+\frac{b^{q}}{q \delta^{\frac{q}{p}}}=\frac{a^{p} \delta}{p}+\frac{b^{q}}{q \delta^{\frac{q}{p}}}, \quad \forall a, b, \delta>0 .
$$

Thus, for any $\delta>0$, we have

$$
\begin{aligned}
\mathbb{E}\left[\sup _{0 \leq t \leq T}|e(t)|^{2}\right] \\
=\mathbb{E}\left[\sup _{0 \leq t \leq T}|e(t)|^{2} I_{\left\{\tau_{d}>T \text { and } \sigma_{d}>T\right\}}\right]+\mathbb{E}\left[\sup _{0 \leq t \leq T}|e(t)|^{2} I_{\left\{\tau_{d} \leq T \text { or } \sigma_{d} \leq T\right\}}\right] \\
=\mathbb{E}\left[\sup _{0 \leq t \leq T}|e(t)|^{2} I_{\left\{v_{d}>T\right\}}\right]+\mathbb{E}\left[\sup _{0 \leq t \leq T}|e(t)|^{2} I_{\left\{\tau_{d} \leq T \text { or } \sigma_{d} \leq T\right\}}\right] \\
\leq \mathbb{E}\left[\sup _{0 \leq t \leq T}\left|e\left(t \wedge v_{d}\right)\right|^{2}\right]+\mathbb{E}\left[\sup _{0 \leq t \leq T}|e(t)|^{2} I_{\left\{\tau_{d} \leq T \text { or } \sigma_{d} \leq T\right\}}\right] \\
\left.\leq \mathbb{E}\left[\sup _{0 \leq t \leq T}\left|e\left(t \wedge v_{d}\right)\right|^{2}\right]+\frac{\delta}{p} \mathbb{E}_{0 \leq t \leq T}|e(t)|^{2 p}\right] \\
\quad+\frac{1-\frac{1}{p}}{\sup ^{\frac{1}{p-1}}} \mathbb{P}\left\{\tau_{d} \leq T \text { or } \sigma_{d} \leq T\right\} .
\end{aligned}
$$

By Lemma 4.2, then

$$
\mathbb{P}\left\{\tau_{d} \leq T\right\} \leq \mathbb{E}\left[I_{\left\{\tau_{d} \leq T\right\}} \frac{\left|X\left(\tau_{d}\right)\right|^{2 p}}{d^{2 p}}\right] \leq \frac{1}{d^{2 p}} \mathbb{E}\left[\sup _{0 \leq t \leq T}|X(t)|^{2 p}\right] \leq \frac{A_{1}}{d^{2 p}}
$$

Similarly, the result can be derived for $\sigma_{d}$

$$
\mathbb{P}\left\{\sigma_{d} \leq T\right\}=\mathbb{E}\left[I_{\left\{\sigma_{d} \leq T\right\}} \frac{\left|\bar{Y}\left(\sigma_{d}\right)\right|^{2 p}}{d^{2 p}}\right] \leq \frac{1}{d^{2 p}} \mathbb{E}\left[\sup _{0 \leq t \leq T}|\bar{Y}(t)|^{2 p}\right] \leq \frac{A_{1}}{d^{2 p}}
$$

so that

$$
\mathbb{P}\left\{\sigma_{d} \leq T \text { or } v_{d} \leq T\right\} \leq \mathbb{P}\left\{\sigma_{d} \leq T\right\}+\mathbb{P}\left\{v_{d} \leq T\right\} \leq \frac{2 A_{1}}{d^{2 p}}
$$


Using the bounds of $X(t)$ and $\bar{Y}(t)$, we have

$$
\mathbb{E}\left[\sup _{0 \leq t \leq T}|e(t)|^{2 p}\right] \leq 2^{2 p-1} \mathbb{E}\left[\sup _{0 \leq t \leq T}\left(|X(t)|^{2 p}+|\bar{Y}(t)|^{2 p}\right)\right] \leq 2^{2 p} A_{1} .
$$

Substituting the above inequality into (4.32) leads to

$$
\begin{aligned}
& \mathbb{E}\left[\sup _{0 \leq t \leq T}|e(t)|^{2}\right] \\
& \quad \leq \mathbb{E}\left[\sup _{0 \leq t \leq T}\left|\bar{Y}\left(t \wedge v_{d}\right)-X\left(t \wedge v_{d}\right)\right|^{2}\right]+\frac{2^{2 p} \delta A_{1}}{p}+\frac{2 A_{1}\left(1-\frac{1}{p}\right)^{2 p}}{d} \delta^{\frac{1}{p-1}} .
\end{aligned}
$$

Now we bound the first term on the right-hand side of (4.33). By the definitions of $X(t)$ and $\bar{Y}(t)$, combining the fact that $0<\theta \leq 1$, we have

$$
\begin{aligned}
\mid \bar{Y}(t & \left.\wedge v_{d}\right)-\left.X\left(t \wedge v_{d}\right)\right|^{2} \\
= & \mid \int_{0}^{t \wedge v_{d}}\left[f_{\lambda}(Y(s))-f_{\lambda}(X(s))\right] \mathrm{d} s \\
& +\int_{0}^{t \wedge v_{d}} g(Y(s))-g(X(s)) \mathrm{d} W(s) \\
& +\int_{0}^{t \wedge v_{d}} h(Y(s))-\left.h(X(s)) \mathrm{d} \tilde{N}(s)\right|^{2} \\
\leq & 3\left|\int_{0}^{t \wedge v_{d}}\left[f_{\lambda}(Y(s))-f_{\lambda}(X(s))\right] \mathrm{d} s\right|^{2} \\
& +3\left|\int_{0}^{t \wedge v_{d}} g(Y(s))-g(X(s)) \mathrm{d} W(s)\right|^{2} \\
& +3\left|\int_{0}^{t \wedge v_{d}} h(Y(s))-h(X(s)) \mathrm{d} \tilde{N}(s)\right|^{2} .
\end{aligned}
$$

For any $\tau \in[0, T]$, using the Cauchy-Schwarz inequality and the Doob martingale inequality, we obtain

$$
\begin{aligned}
& \mathbb{E}\left[\sup _{0 \leq t \leq \tau}\left|\bar{Y}\left(t \wedge v_{d}\right)-X\left(t \wedge v_{d}\right)\right|^{2}\right] \\
& \leq 3 T \mathbb{E} \int_{0}^{\tau \wedge v_{d}}\left|f_{\lambda}(Y(s))-f_{\lambda}(X(s))\right|^{2} \mathrm{~d} s \\
& \quad+12 \mathbb{E} \int_{0}^{\tau \wedge v_{d}}|g(Y(s))-g(X(s))|^{2} \mathrm{~d} s \\
& \quad+12 \mathbb{E} \lambda \int_{0}^{\tau \wedge v_{d}}|h(Y(s))-h(X(s))|^{2} \mathrm{~d} s .
\end{aligned}
$$

Applying the local Lipschitz condition (4.30) and Assumption 2.1, we get

$$
\begin{aligned}
\mathbb{E} & {\left[\sup _{0 \leq t \leq \tau}\left|\bar{Y}\left(t \wedge v_{d}\right)-X\left(t \wedge v_{d}\right)\right|^{2}\right] } \\
& \leq\left(3 T L_{R}+12 L_{g}+12 L_{h} \lambda\right) \mathbb{E} \int_{0}^{\tau \wedge v_{d}}|Y(s)-X(s)|^{2} \mathrm{~d} s
\end{aligned}
$$




$$
\begin{aligned}
\leq & 2\left(3 T L_{R}+12 L_{g}+12 L_{h} \lambda\right)\left[\mathbb{E} \int_{0}^{\tau \wedge v_{d}}|Y(s)-\bar{Y}(s)|^{2} \mathrm{~d} s\right. \\
& \left.+\int_{0}^{\tau} \mathbb{E} \sup _{0 \leq r \leq s}\left|\bar{Y}\left(r \wedge v_{d}\right)-X\left(r \wedge v_{d}\right)\right|^{2} \mathrm{~d} s\right] .
\end{aligned}
$$

To bound the first term inside the parentheses of (4.34), we denote by $n_{s}$ the integer for which $s \in\left[t_{n_{s}}, t_{n_{s+1}}\right]$ and note that

$$
\begin{aligned}
Y(s)-\bar{Y}(s)= & -f_{\lambda}\left(Y_{n_{s}}^{*}\right)\left(s-t_{n_{s}}\right)-g\left(Y_{n_{s}}^{*}\right)\left(W(s)-W\left(t_{n_{s}}\right)\right) \\
& -h\left(Y_{n_{s}}^{*}\right)\left(\tilde{N}(s)-\tilde{N}\left(t_{n_{s}}\right)\right),
\end{aligned}
$$

and hence that

$$
|Y(s)-\bar{Y}(s)|^{2} \leq 3\left[\left|f_{\lambda}\left(Y_{n_{s}}^{*}\right) \Delta t\right|^{2}+\left|g\left(Y_{n_{s}}^{*}\right) \Delta W_{n_{s}}\right|^{2}+\left|h\left(Y_{n_{s}}^{*}\right) \Delta N_{n_{s}}\right|^{2}\right]
$$

Note that

$$
\begin{aligned}
\left|f_{\lambda}\left(Y_{n_{s}}^{*}\right)\right|^{2} & \leq 2\left[\left|f_{\lambda}\left(Y_{n_{s}}^{*}\right)-f_{\lambda}(0)\right|^{2}+\left|f_{\lambda}(0)\right|^{2}\right] \\
& \leq 2 L_{R}\left|Y_{n_{s}}^{*}\right|^{2}+2\left|f_{\lambda}(0)\right|^{2} .
\end{aligned}
$$

Thus by the second moments of martingale increments and the moment bound on the numerical solution $Y_{n}^{*}$, we can obtain

$$
\mathbb{E} \int_{0}^{\tau \wedge v_{d}}|Y(s)-\bar{Y}(s)|^{2} \mathrm{~d} s \leq C_{1} \Delta t
$$

for a constant $C_{1}=C_{1}(R, T, A)$. Substituting this bound into (4.34) and applying the continuous Gronwall inequality gives

$$
\mathbb{E}\left[\sup _{0 \leq t \leq T}\left|\bar{Y}\left(t \wedge v_{d}\right)-X\left(t \wedge v_{d}\right)\right|^{2}\right] \leq C_{2} \Delta t e^{\left(3 T L_{R}+12 L_{g}+12 L_{h} \lambda\right) T},
$$

for a constant $C_{2}=C_{2}(R, T, A)$.

Now combining (4.35) with (4.33), we have

$$
\mathbb{E}\left[\sup _{0 \leq t \leq T}|e(t)|^{2}\right] \leq C_{2} \Delta t e^{\left(3 T L_{R}+12 L_{g}+12 L_{h} \lambda\right) T}+\frac{2^{2 p} \delta A_{1}}{p}+\frac{2 A_{1}\left(1-\frac{1}{p}\right)^{2 p}}{d} \delta^{\frac{1}{p-1}} .
$$

For any given $\varepsilon>0$, we can choose $\delta$ sufficiently small for

$$
\frac{2^{2 p} \delta A_{1}}{p} \leq \frac{\varepsilon}{3}
$$

and then choose $d$ sufficient large for

$$
\frac{2 A_{1}\left(1-\frac{1}{p}\right)}{d^{2 p} \delta^{\frac{1}{p-1}}}<\frac{\varepsilon}{3}
$$


and finally choose $\Delta t$ so that

$$
C_{2} \Delta t e^{\left(3 T L_{R}+12 L_{g}+12 L_{h} \lambda\right) T}<\frac{\varepsilon}{3}
$$

Thus $\mathbb{E}\left[\sup _{0 \leq t \leq T}|e(t)|^{2}\right]<\varepsilon$. The proof is completed.

\section{Convergence rate}

To prove the convergence rate of the $\operatorname{CSS} \theta$ method, we give the following assumption.

Assumption 5.1 There exist constants $D \in \mathbb{R}^{+}$and $q \in \mathbb{Z}^{+}$such that, for all $a, b \in \mathbb{R}^{n}$,

$$
\left|f_{\lambda}(a)-f_{\lambda}(b)\right|^{2} \leq D\left(1+|a|^{q}+|b|^{q}\right)|a-b|^{2} .
$$

Firstly, we establish Lemma 5.1 under Assumptions 2.1 and 5.1.

Lemma 5.1 Under Assumptions 2.1 and 5.1, let $0<\theta \leq 1,0<\Delta t<\min \left\{1, \frac{1}{2 \theta L_{\lambda}}\right\}$, for any given integer $r \geq 2$, there exists a positive constant $E=E(r)$ such that

$$
\mathbb{E} \sup _{0 \leq t \leq T}|Y(t)-\bar{Y}(t)|^{r} \leq E \Delta t^{\frac{r}{2}}
$$

Proof Since for any given $t \in[n \Delta t,(n+1) \Delta t]$, we have $Y(t)=Y_{n}$, and then by the continuous-time approximate solution $\bar{Y}(t)$ defined by (4.3), we can get

$$
\begin{aligned}
Y(t)-\bar{Y}(t)= & -f_{\lambda}\left(Y_{n}^{*}\right)\left(t-t_{n}\right)-g\left(Y_{n}^{*}\right)\left(W(t)-W\left(t_{n}\right)\right) \\
& -h\left(Y_{n}^{*}\right)\left(\tilde{N}(t)-\tilde{N}\left(t_{n}\right)\right),
\end{aligned}
$$

and hence for $t-t_{n} \leq \Delta t$

$$
\begin{aligned}
|Y(t)-\bar{Y}(t)|^{r} \leq & 3^{r-1}\left[\Delta t^{r}\left|f_{\lambda}\left(Y_{n}^{*}\right)\right|^{r}+\left|g\left(Y_{n}^{*}\right)\right|^{r}\left|W(t)-W\left(t_{n}\right)\right|^{r}\right. \\
& \left.+\left|h\left(Y_{n}^{*}\right)\right|^{r}\left|\tilde{N}(t)-\tilde{N}\left(t_{n}\right)\right|^{r}\right] .
\end{aligned}
$$

By Assumption 5.1 on $f_{\lambda}$, and linear growth condition (2.6)-(2.7) for $g$ and $h$, we have

$$
\begin{aligned}
\mathbb{E} \sup _{0 \leq t \leq T}|Y(t)-\bar{Y}(t)|^{r} \\
\leq C_{3}(r)\left[\Delta t^{r}\left(1+\sup _{0 \leq t \leq T} \mathbb{E}\left|Y_{n}^{*}\right|^{u}\right)+\left(1+\sup _{0 \leq t \leq T} \mathbb{E}\left|Y_{n}^{*}\right|^{u}\right)\left|t-t_{n}\right|^{r / 2}\right. \\
\left.\quad+\left(1+\sup _{0 \leq t \leq T} \mathbb{E}\left|Y_{n}^{*}\right|^{u}\right)\left|t-t_{n}\right|^{r / 2}\right],
\end{aligned}
$$

where $C_{3}(r)$ and $u$ are both integer constants depending on $q$ from Assumption 5.1 and $r$. By Lemma 4.1, we obtain

$$
\mathbb{E} \sup _{0 \leq t \leq T}|Y(t)-\bar{Y}(t)|^{r} \leq E \Delta t^{\frac{r}{2}}
$$

where $E=E(r)$ a positive constant which depends on $r$. 
Theorem 5.2 Under Assumptions 2.1 and 5.1, let $0<\theta \leq 1,0<\Delta t<\min \left\{1, \frac{1}{2 \theta L_{\lambda}}\right\}$, the continuous-time approximate solution $\bar{Y}(t)$ defined by (4.3) will converge to the true solution $X(t)$ of (3.1) with strong order of one half, i.e.

$$
\mathbb{E} \sup _{0 \leq t \leq T}|\bar{Y}(t)-X(t)|^{2}=O(\Delta t)
$$

Proof Let

$$
e(t)=\bar{Y}(t)-X(t)
$$

From the identity

$$
\begin{aligned}
X(t)= & X_{0}+\int_{0}^{t} f_{\lambda}\left(X\left(s^{-}\right)\right) \mathrm{d} s+\int_{0}^{t} g\left(X\left(s^{-}\right)\right) \mathrm{d} W(s) \\
& +\int_{0}^{t} h\left(X\left(s^{-}\right)\right) \mathrm{d} \tilde{N}(s),
\end{aligned}
$$

and (4.3), we apply the Itô formula [17] to obtain

$$
\begin{aligned}
|e(t)|^{2}= & 2 \int_{0}^{t}\left\langle f_{\lambda}\left(Y\left(s^{-}\right)\right)-f_{\lambda}\left(X\left(s^{-}\right)\right), e\left(s^{-}\right)\left|\mathrm{d} s+\int_{0}^{t}\right| g\left(Y\left(s^{-}\right)\right)-\left.g\left(X\left(s^{-}\right)\right)\right|^{2} \mathrm{~d} s\right. \\
& +\lambda \int_{0}^{t}\left|h\left(Y\left(s^{-}\right)\right)-h\left(X\left(s^{-}\right)\right)\right|^{2} \mathrm{~d} s \\
& +\int_{0}^{t} 2\left\langle e\left(s^{-}\right), g\left(Y\left(s^{-}\right)\right)-g\left(X\left(s^{-}\right)\right) \mathrm{d} W(s)\right\rangle \\
& +\int_{0}^{t} 2\left\langle e\left(s^{-}\right), h\left(Y\left(s^{-}\right)\right)-h\left(X\left(s^{-}\right)\right)\right\rangle \mathrm{d} \tilde{N}(s) \\
& +\int_{0}^{t}\left|h\left(Y\left(s^{-}\right)\right)-h\left(X\left(s^{-}\right)\right)\right|^{2} \mathrm{~d} \tilde{N}(s) \\
\leq & 2 \int_{0}^{t}\left\langle f_{\lambda}\left(Y\left(s^{-}\right)\right)-f_{\lambda}\left(\bar{Y}\left(s^{-}\right)\right), e\left(s^{-}\right)\right\rangle+\left\langle f_{\lambda}\left(\bar{Y}\left(s^{-}\right)\right)-f_{\lambda}\left(X\left(s^{-}\right)\right), e\left(s^{-}\right)\right\rangle \mathrm{d} s \\
& +\int_{0}^{t}\left|g\left(Y\left(s^{-}\right)\right)-g\left(X\left(s^{-}\right)\right)\right|^{2} \mathrm{~d} s \\
& +\lambda \int_{0}^{t}\left|h\left(Y\left(s^{-}\right)\right)-h\left(X\left(s^{-}\right)\right)\right|^{2} \mathrm{~d} s \\
& +M_{1}(t)+M_{2}(t)+M_{3}(t),
\end{aligned}
$$

where

$$
\begin{aligned}
& M_{1}(t)=\int_{0}^{t} 2\left\langle e\left(s^{-}\right), g\left(Y\left(s^{-}\right)\right)-g\left(X\left(s^{-}\right)\right) \mathrm{d} W(s)\right\rangle, \\
& M_{2}(t)=\int_{0}^{t} 2\left\langle e\left(s^{-}\right), h\left(Y\left(s^{-}\right)\right)-h\left(X\left(s^{-}\right)\right)\right\rangle \mathrm{d} \tilde{N}(s), \\
& M_{3}(t)=\int_{0}^{t}\left|h\left(Y\left(s^{-}\right)\right)-h\left(X\left(s^{-}\right)\right)\right|^{2} \mathrm{~d} \tilde{N}(s) .
\end{aligned}
$$


Using the Assumptions 2.1 and 5.1, and (3.2) we have

$$
\begin{aligned}
& |e(t)|^{2} \leq \int_{0}^{t} 2\left\langle f_{\lambda}\left(Y\left(s^{-}\right)\right)-f_{\lambda}\left(\bar{Y}\left(s^{-}\right)\right), e\left(s^{-}\right)\right\rangle+2 K_{\lambda}\left|e\left(s^{-}\right)\right|^{2} \mathrm{~d} s \\
& +\int_{0}^{t}\left(L_{g}+\lambda L_{h}\right)\left|Y\left(s^{-}\right)-X\left(s^{-}\right)\right|^{2} \mathrm{~d} s \\
& +M_{1}(t)+M_{2}(t)+M_{3}(t) \\
& \leq \int_{0}^{t}\left|f_{\lambda}\left(Y\left(s^{-}\right)\right)-f_{\lambda}\left(\bar{Y}\left(s^{-}\right)\right)\right|^{2}+\left|e\left(s^{-}\right)\right|^{2} \mathrm{~d} s+2 K_{\lambda} \int_{0}^{t}\left|e\left(s^{-}\right)\right|^{2} \mathrm{~d} s \\
& +2\left(L_{g}+\lambda L_{h}\right) \int_{0}^{t}\left|e\left(s^{-}\right)\right|^{2}+\left|Y\left(s^{-}\right)-\bar{Y}\left(s^{-}\right)\right|^{2} \mathrm{~d} s \\
& +M_{1}(t)+M_{2}(t)+M_{3}(t) \\
& \leq\left[1+2\left(K_{\lambda}+L_{g}+\lambda L_{h}\right)\right] \int_{0}^{t}\left|e\left(s^{-}\right)\right|^{2} \mathrm{~d} s \\
& +D \int_{0}^{t}\left(1+\left|Y\left(s^{-}\right)\right|^{q}+\left|\bar{Y}\left(s^{-}\right)\right|^{q}\right)\left|Y\left(s^{-}\right)-\bar{Y}\left(s^{-}\right)\right|^{2} \mathrm{~d} s \\
& +2\left(L_{g}+\lambda L_{h}\right) \int_{0}^{t}\left|Y\left(s^{-}\right)-\bar{Y}\left(s^{-}\right)\right|^{2} \mathrm{~d} s \\
& +M_{1}(t)+M_{2}(t)+M_{3}(t) \\
& \leq\left[1+2\left(K_{\lambda}+L_{g}+\lambda L_{h}\right)\right] \int_{0}^{t}\left|e\left(s^{-}\right)\right|^{2} \mathrm{~d} s \\
& +D_{1}\left(\sup _{0 \leq s \leq t}\left|Y\left(s^{-}\right)-\bar{Y}\left(s^{-}\right)\right|^{2}\right) \int_{0}^{t}\left(1+\left|Y\left(s^{-}\right)\right|^{q}+\left|\bar{Y}\left(s^{-}\right)\right|^{q}\right) \mathrm{d} s \\
& +M_{1}(t)+M_{2}(t)+M_{3}(t),
\end{aligned}
$$

where we use $D_{1}$ to denote a generic constant (independent of $\Delta t$ ) that may change from line to line.

Using the Lemma 4.1, Lemma 4.2 and Lemma 5.1, we have

$$
\begin{aligned}
\mathbb{E} \sup _{0 \leq s \leq t}|e(s)|^{2} \leq & D_{1} \int_{0}^{t} \mathbb{E}\left|e\left(s^{-}\right)\right|^{2} \mathrm{~d} s+D_{1} \Delta t \\
& +\mathbb{E} \sup _{0 \leq s \leq t} M_{1}(s)+\mathbb{E} \sup _{0 \leq s \leq t} M_{2}(s)+\mathbb{E} \sup _{0 \leq s \leq t} M_{3}(s) .
\end{aligned}
$$

Now, as in the proof of [9], the Burkholder-Davis-Gundy inequality can be used to get the estimate

$$
\mathbb{E} \sup _{0 \leq s \leq t} M_{i}(s) \leq \frac{1}{2} \mathbb{E} \sup _{0 \leq s \leq t}|e(t)|^{2}+D_{1} \int_{0}^{t} \mathbb{E}\left|e\left(s^{-}\right)\right|^{2} \mathrm{~d} s+D_{1} \Delta t, \quad i=1,2,3 .
$$

Using this in (5.6), we obtain

$$
\mathbb{E} \sup _{0 \leq s \leq t}|e(s)|^{2} \leq D_{1} \int_{0}^{t} \mathbb{E} \sup _{0 \leq s \leq t}\left|e\left(s^{-}\right)\right|^{2} \mathrm{~d} s+D_{1} \Delta t .
$$

The result follows from the continuous Gronwall inequality. 


\section{Numerical experiments}

We consider the following nonlinear stochastic different equation with jumps from [16]:

$$
\left\{\begin{array}{l}
\mathrm{d} X(t)=\left(-4 X\left(t^{-}\right)-X^{3}\left(t^{-}\right)\right) \mathrm{d} t+X\left(t^{-}\right) \mathrm{d} W(t)+X\left(t^{-}\right) \mathrm{d} N(t) \\
X(0)=1
\end{array}\right.
$$

Define $f(x(t))=-4 x(t)-x^{3}(t), g(x(t))=x(t), h(x(t))=x(t)$. It is easy to compute that

$$
\begin{aligned}
\langle x-y, f(x)-f(y)\rangle & =\left\langle x-y,-4(x-y)-\left(x^{3}-y^{3}\right)\right\rangle \\
& =-4|x-y|^{2}\left(1+x^{2}+x y+y^{2}\right) \\
& \leq-4|x-y|^{2},
\end{aligned}
$$

which implies that $f(x)$ satisfies the one-sided Lipschitz condition, $g(x)$ and $h(x)$ satisfy the global Lipschitz condition, then the Assumptions of Theorem 5.2 hold. That is to say, the numerical solution by our method will converge to the true solution of system (6.1).

To show the convergence of the CSS $\theta$ method for system (6.1), we fix $\Delta t=2^{-14}, T=2$, $\lambda=1, \theta=0.7$. Noting that the exact solution of nonlinear jump-diffusion system (6.1) is not available, we use the numerical solution by the SSBE method with step size $\Delta t=2^{-14}$ as the 'referenced exact solution' (Theorem 2 in [16] can guarantee its strong convergence) in Figure 1.

In Figure 1, we show the numerical solution by the $\operatorname{CSS} \theta$ method with step size $\Delta t=2^{-10}$ and the 'referenced exact solution. we can easy to find that the $\operatorname{CSS} \theta$ approximation and the 'referenced exact solution' make no major difference between both paths. That is to say the CSS $\theta$ method converges to the 'referenced exact solution' well. Hence our method is efficient for the nonlinear jump-diffusion systems.

To show the strong convergence order of the $\operatorname{CSS} \theta$ method with different parameter $\theta$, we fix $T=2, \lambda=1$ and change $\theta=0.5,0.7,0.9$, respectively. The 'referenced exact solution' of system (6.1) is also used by the SSBE method with step size $\Delta t=2^{-14}$. We simulate the numerical solutions with five different step sizes $h=2^{p-1} \Delta t$ for $1 \leq p \leq 5, \Delta t=2^{-14}$. The mean-square errors $\varepsilon=1 / 5,000 \sum_{i=1}^{5,000}\left|Y_{n}\left(\omega_{i}\right)-X(T)\right|^{2}$, all measured at time $T=2$, are estimated by trajectory averaging. We plot our approximation to $\sqrt{\epsilon}$ against $\Delta t$ on a log$\log$ scale. For reference a dashed line of slope one-half is added.

In Figure 2, we see that the slopes of the four curves appear to match well. Therefore, the results verify the strong convergence order of the proposed method.

Figure $1 \operatorname{CSS} \theta$ solution and 'referenced exact solution' for system (6.1).

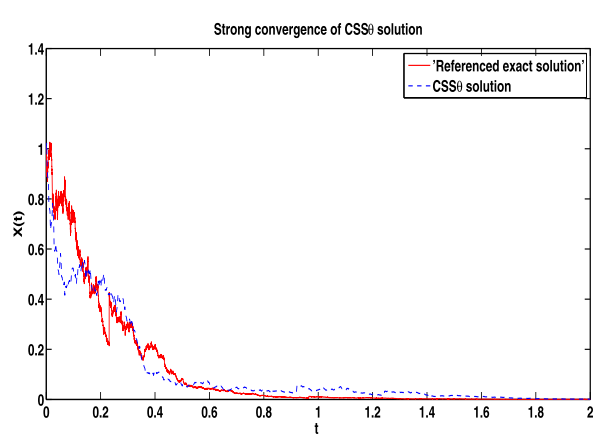




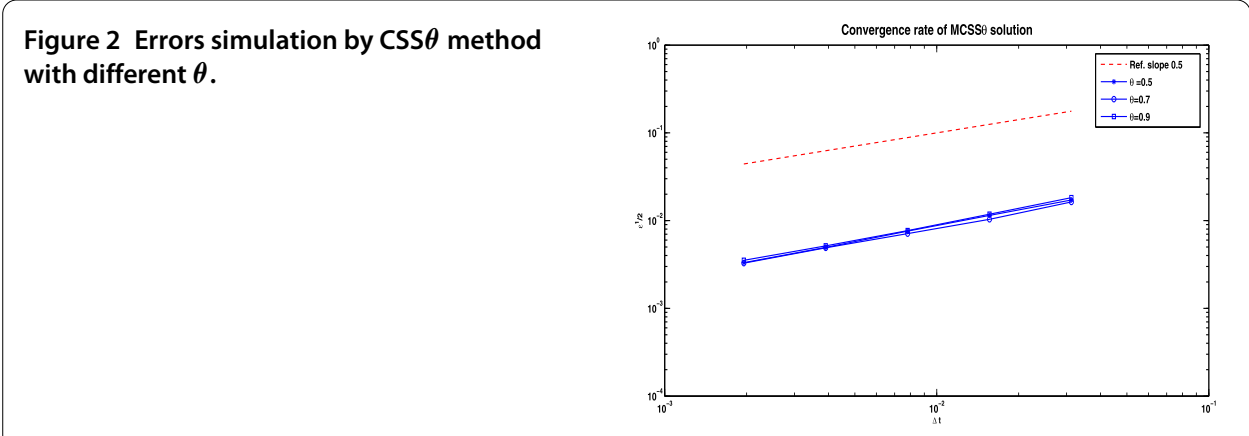

\section{Acknowledgements}

This research is partial supported with funds provided by the National Natural Science Foundation of China (Grant Nos. 11501410, 11471243 and 11672207), and in part by Natural Science Foundation of Tianjin (No: 17JCQNJC03800). We thank the anonymous reviewers for their very valuable comments and helpful suggestions which improve this paper significantly.

\section{Competing interests}

The authors declare that they have no competing interests.

\section{Authors' contributions}

All the authors contributed equally to this work. They all read and approved the final version of the manuscript.

\section{Author details}

${ }^{1}$ Department of Mathematics, Tianjin Polytechnic University, Tianjin, 300387, P.R. China. ${ }^{2}$ Department of Sport Culture and Communication, Tianjin University of Sport, Tianjin, 300381, P.R. China.

\section{Publisher's Note}

Springer Nature remains neutral with regard to jurisdictional claims in published maps and institutional affiliations.

Received: 15 August 2016 Accepted: 16 June 2017 Published online: 30 June 2017

\section{References}

1. Bruti-Liberati, N, Platen, E: Strong approximations of stochastic differential equations with jumps. J. Comput. Appl. Math. 205, 982-1001 (2007)

2. Chalmers, GD, Higham, DJ: Convergence and stability analysis for implicit simulations of stochastic differentia equations with random jump magnitudes. Discrete Contin. Dyn. Syst., Ser. B 9, 47-64 (2008)

3. Higham, DJ, Kloeden, PE: Convergence and stability of implicit methods for jump-diffusion. Int. J. Numer. Anal. Model. 3, 125-140 (2006)

4. Hu, L, Gan, SQ: Convergence and stability of the balanced methods for stochastic differential equations with jumps. Int. J. Comput. Math. 88, 2089-2108 (2011)

5. Tan, JG, Mu, ZM, Guo, YF: Convergence and stability of the compensated split-step $\theta$-method for stochastic differential equations with jumps. Adv. Differ. Equ. 2014, 209 (2014)

6. Wang, XJ, Gan, SQ: Compensated stochastic theta methods for stochastic differential equations with jumps. Appl. Numer. Math. 60, 87-887 (2010)

7. Jiang, F, Shen, Y, Wu, FK: Jump systems with the mean-reverting $\gamma$-process and convergence of the numerical approximation. Stoch. Dyn. 12, 1150018 (2012)

8. Bao, J, Mao, XR, Yin, G: Competitive Lotka-Volterra population dynamics with jumps. Nonlinear Anal. 74, 6601-6616 (2011)

9. Higham, DJ, Mao, XR, Stuart, AM: Strong convergence of Euler-type methods for nonlinear stochastic differential equations. SIAM J. Numer. Anal. 40, 1041-1063 (2002)

10. Hutzenthaler, M, Jentzen, A, Kloeden, PE: Strong and weak divergence in finite time of Euler's method for stochastic differential equations with non-globally Lipschitz continuous coefficients. Proc. R. Soc. Lond., Ser. A, Math. Phys. Eng. Sci. 467, 1563-1576 (2010)

11. Mao, XR, Szpruch, L: Strong convergence and stability of implicit numerical methods for stochastic differential equations with non-globally Lipschitz continuous coefficients. J. Comput. Appl. Math. 238, 14-28 (2013)

12. Mao, XR, Szpruch, L: Strong convergence rates for backward Euler-Maruyama method for non-linear dissipative-type stochastic differential equations with super-linear diffusion coefficients. Stochastics 85, 144-171 (2013)

13. Huang, CM: Exponential mean square stability of numerical methods for systems of stochastic differential equations. J. Comput. Appl. Math. 236, 4016-4026 (2012)

14. Zong, XF, Wu, FK, Huang, CM: Exponential mean square stability of the theta approximations for neutral stochastic differential delay equations. J. Comput. Appl. Math. 286, 172-185 (2015)

15. Zong, XF, Wu, FK, Huang, CM: Theta schemes for SDDEs with non-globally Lipschitz continuous coefficients. J. Comput. Appl. Math. 278, 258-277 (2015)

16. Higham, DJ, Kloeden, PE: Numerical methods for nonlinear stochastic differential equations with jumps. Numer. Math. 101, 101-119(2005) 
17. Higham, DJ, Kloeden, PE: Strong convergence rates for backward Euler on a class of nonlinear jump-diffusion problems. J. Comput. Appl. Math. 205, 949-956 (2007)

18. Gyöngy, I, Krylov, NV: On stochastic equations with respect to semi-martingales I. Stochastics 4, 1-21 (1980)

Submit your manuscript to a SpringerOpen ${ }^{\circ}$ journal and benefit from:

- Convenient online submission

$\checkmark$ Rigorous peer review

- Open access: articles freely available online

- High visibility within the field

Retaining the copyright to your article

Submit your next manuscript at $\boldsymbol{\nabla}$ springeropen.com 